designed to include concepts typically conveyed during inclinic HIV counselling sessions (e.g., window period, public health reporting). The aim of this study was to compare knowledge of key HIV test concepts between clients testing through GCO and in-clinic.

Methods GCO and clinic participants were concurrently recruited over 11 months. Participants were invited to complete an anonymous online survey 2 weeks after receipt of test results. Knowledge of key concepts related to HIV testing was measured using a 6-item true/false test previously developed through a modified Delphi process, cognitive testing and psychometric evaluation. Linear regression was used to assess the association between site (GCO vs. clinic) and overall test scores, after adjustment for age, education, immigration history, language, sexual orientation, and testing history.

Results 404 HIV-negative participants were included in the analysis (73 GCO, 331 in-clinic). HIV test knowledge scores averaged 0.4 points higher among GCO (mean score 4.5) than among clinic $(4.1)$ testers $(p=0.01)$. Following adjustment for relevant covariates, this difference decreased to 0.2 points $(p=0.15)$. Likewise, there was no difference in mean HIV test knowledge scores among first-time testers $(n=50 ; 3.7$ GCO, 3.6 in-clinic; $\mathrm{p}=0.75$ ).

Conclusion Post-test knowledge of HIV test concepts addressed in standard pre-test counselling was high in both groups and not significantly different following adjustment. Our study suggests that equivalent education about core HIV testing concepts can be achieved through web-based HIV/STI testing, and illustrates the importance of designing services to intentionally address relevant educational messages covered in providerdelivered HIV test counselling.

\section{P2.45 HIGH BURDEN OF PERSISTENT ONCOGENIC HPV INFECTION IN HIGH-RISK, HIV-NEGATIVE MEN WHO HAVE SEX WITH MEN USING A NOVEL HPV E6/E7 MRNA ASSAY}

${ }^{1}$ Troy Grennan, ${ }^{2}$ Marek Smieja, ${ }^{2}$ Max Chernesky, ${ }^{1}$ Ronita Nath, ${ }^{2}$ Dan Jang, ${ }^{3}$ Alex Schnubb, ${ }^{3}$ Darrell H Tan. ${ }^{1} B C$ Centre for Disease Control, Vancouver, Canada; ${ }^{2} S t$. Joseph's Healthcare, Hamilton, Canada; 'St. Michael's Hospital, Toronto, Canada

10.1136/sextrans-2017-053264.221

Introduction Persistent infection with high-risk human papillomavirus (hrHPV) is a necessary step in anal cancer's pathogenesis. With no universally-accepted guidelines on screening, and given the suboptimal performance of cytology, anal HPV testing is increasingly recognised as an important, adjunctive screening tool for anal cancer precursors. mRNA-based HPV assays targeting the E6/7 oncogenes are emerging as more specific tests for persistent HPV than the traditionally-used DNAbased tests. No data exists on serial monitoring of anal hrHPV in MSM using this novel assay. The objectives of this study are to describe HPV prevalence/persistence rates in a sample of high-risk, HIV-negative MSM enrolled in a PrEP demonstration project.

Methods Participants were drawn from PREPARATORY-5, which recruited HIV-negative MSM with high HIV risk as determined by a score of $\geq 10$ on the HIV Incidence Risk Index for MSM (HIRI-MSM) and a history of condomless receptive anal sex in the prior 6 months. Anal samples were tested for hrHPV via the mRNA-based Aptima HPV Assay at baseline, months 6 and 12. Logistic regression was used to assess characteristics associated with hrHPV infection.

Results 43 participants were recruited, with median age 33 years (IQR 28-37). 10 (23\%) were current smokers, and median HIRI-MSM score was 28 (IQR 19.5-35.5). 24 (56\%) participants had hrHPV at any timepoint, and 15 (35\%) had persistent hrHPV, defined as hrHPV isolated at two different timepoints. In multivariable logistic regression, current smoking status $(\mathrm{OR}=9.2, \quad 95 \% \mathrm{CI}=1.16-72.59, \mathrm{p}=0.03)$ and HIRIMSM score (OR: 1.2 per 1-point increase, 95\% $\mathrm{CI}=1.03-1.33$, $\mathrm{p}=0.01$ ) were associated with hrHPV infection.

Conclusion Using a novel HPV E6/E7 mRNA assay with higher specificity for persistent infection, this study demonstrated a high burden of overall and persistent hrHPV infection in high-risk, HIV-negative MSM. These findings support the inclusion of MSM at high risk of sexual HIV acquisition when considering interventions related to the prevention and screening of anal cancer precursors.

\section{P2.46 EVALUATION OF EFFECTIVENESS AND SAFETY OF ACYCLOVIR 1 GM ONCE A DAY SINGLE ORAL DOSE FOR 5 DAYS FOR TREATMENT OF GENITAL HERPES}

Verma Kaushal, M Sonune, S Gupta. All India Institute of Medical Sciences, New Delhi, India

\subsection{6/sextrans-2017-053264.222}

Introduction Recurrent genital herpes is conventionally treated with acyclovir $200 \mathrm{mg} 5$ times a day orally which is inconvenient to take by the patients. We studied the effectiveness and safety of acyclovir $1 \mathrm{gm}$ single oral dose once a day for 5 days in treatment of recurrent genital herpes.

Methods Patients presenting with recurrent genital herpes were included in the study. After a complete clinical and laboratory evaluation, the patients were treated with acyclovir $1 \mathrm{gm}$ single oral dose once a day for 5 days and followed up on days 5,7 and 10 to determine the response to treatment and adverse effects.

Results There were 19 patients (18 males and 1 female, between 18-47 years of age; mean age: 33.52 \pm 8.09 years), of which 18 patients completed the study. Seven (39\%) of them had complete healing of the ulcer on day 5, $13(72 \%)$ on day 7 and $16(89 \%)$ by day 10 . Mean percentage healing of ulcer was $72.53 \pm 22.24,87.73 \pm 15.22$ and $95.00 \pm 7.07$ on day 5,7 and 10 respectively. Visual analogue score (VAS) showed complete improvement in VAS in $9(50 \%)$ patients on day 5,13 (72.22\%) on day 7 and $17(94.5 \%)$ on day 10 . The mean time of complete improvement in VAS was $6.38 \pm 2.97$ days. Mean of percentage improvement in VAS was $81.92 \pm 20.87$ on day $5,90.00 \pm 15.18$ on day 7 and $92.00 \pm 17.88$ on day 10. Mean healing time of the lesions was $6.86 \pm 2.67$ days (range 3-12 days). There were no significant adverse effects of the therapy.

Conclusion The study demonstrated that oral acyclovir $1 \mathrm{gm}$ once a day as single daily dose is effective and safe for the treatment of recurrent genital herpes. There was significant healing of lesions, which reduces morbidity, psychological distress and risk of transmission of infections to sexual partner. Further studies are however needed to confirm our results. 